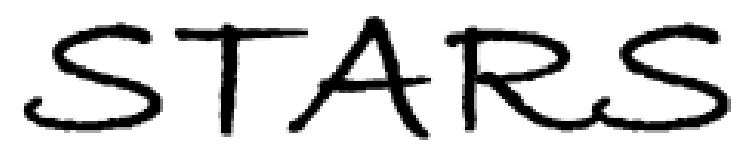

University of Central Florida

STARS

$1-1-1999$

\title{
Histogram specification of 24-bit color images in the color difference $(C-Y)$ color space
}

\author{
A. R. Weeks \\ University of Central Florida \\ L. J. Sartor \\ H. R. Myler \\ University of Central Florida
}

Find similar works at: https://stars.library.ucf.edu/facultybib1990

University of Central Florida Libraries http://library.ucf.edu

This Article is brought to you for free and open access by the Faculty Bibliography at STARS. It has been accepted for inclusion in Faculty Bibliography 1990s by an authorized administrator of STARS. For more information, please contact STARS@ucf.edu.

\section{Recommended Citation}

Weeks, A. R.; Sartor, L. J.; and Myler, H. R., "Histogram specification of 24-bit color images in the color difference (C-Y) color space" (1999). Faculty Bibliography 1990s. 2889.

https://stars.library.ucf.edu/facultybib1990/2889

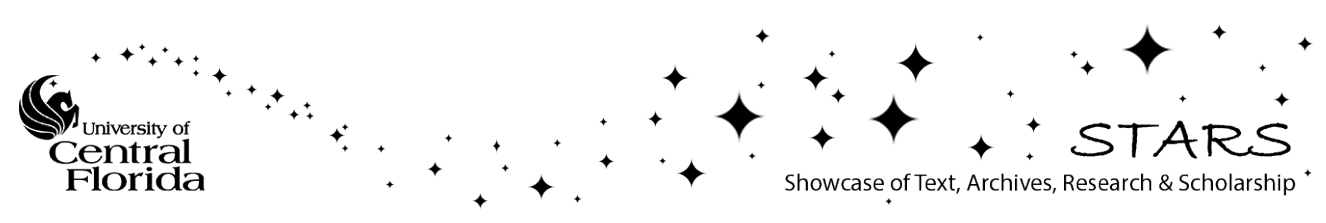




\section{Histogram specification of 24-bit color images in the color difference $(\mathrm{C}-\mathrm{Y})$ color space}

Arthur Robert Weeks, Lloyd J. Sartor, Harley R. Myler

Arthur Robert Weeks, Lloyd J. Sartor, Harley R. Myler, "Histogram specification of 24-bit color images in the color difference (C-Y) color space," Proc. SPIE 3646, Nonlinear Image Processing X, (5 March 1999); doi: $10.1117 / 12.341098$

SPIE. Event: Electronic Imaging '99, 1999, San Jose, CA, United States 


\title{
Histogram Specification of 24-bit color images in the color difference (C-Y) color space
}

\author{
A. R. Weeks', L. J. Sartor ${ }^{2}$, and H. R. Myler ${ }^{1}$ \\ 1: Department of Electrical and Computer Engineering \\ University of Central Florida \\ Orlando, Florida 32816-0450 \\ 2: Siemens Telecom Networks \\ 400 Rinehart Road \\ Lake Mary, Florida 32708
}

\begin{abstract}
Histogram equalization and specification have been widely used to enhance the content of grayscale images, with histogram specification having the advantage of allowing the output histogram to be specified as compared to histogram equalization which attempts to produce and output histogram which is uniform. Unfortunately, extending histogram techniques to color images is not very straightforward. Performing histogram specification on color images in the RGB color space results in specified histograms that are hard to interpret for a particular enhancement that is desired. Human perception of color interprets a color in terms of its hue, saturation, and intensity components. In this paper, we describe a method of extending graylevel histogram specification to color images by performing histogram specification on the luminance (or intensity), saturation, and hue components in the color difference (C-Y) color space. This method takes into account the correlation between the hue, saturation, and intensity components while yielding specified histograms which have physical meaning. Histogram specification was performed on an example color image and was shown to enhance the color content and details within this image without introducing unwanted artifacts.
\end{abstract}

Keywords: Color Enhancement, Histogram Techniques, Histogram Specification, Color Image Processing

\section{INTRODUCTION}

While grayscale image processing has become quite a mature field, much less work has been done with regard to color image processing. Only recently has the hardware for processing high-resolution 24 -bit ( 8 bits per color component) color images become generally available and affordable. Presently, there exists video cards, digitizers, scanners, and printers that manipulate 24-bit color images. Besides color photography and color video processing, many other applications from satellite imaging to multi-spectral biomedical image processing can benefit from color image processing techniques. Many publications have appeared recently that focus on the enhancement of color images by extending grayscale image processing techniques to color images. $1,2,3$ Unfortunately, the extension from grayscale image processing to color image processing is not trivial. There exists information between the different color components that must be maintained during enhancement or restoration. Attempts have been made to simply apply grayscale image processing methods to color images by applying the grayscale algorithm to each of the color components independently. Unfortunately, this approach yields unsatisfactory results. Other color image processing approaches avoid this issue by processing only the intensity aspect of color images. While effective, this approach ignores the benefits of using the color information in conjunction with the intensity information.

Histogram equalization is a well understood technique for enhancing graylevel images. 3,4 The goal of histogram equalization is to redistribute the graylevels within an image producing a new graylevel distribution which is approximately uniform. Histogram equalization methods have been successfully used to improve the overall brightness and contrast of an image, enhancing features that otherwise would not be observable. In addition, histogram equalization provides a means in 
which images with different brightness and contrast values can easily be compared. Comparing features within images that contain drastically different brightness and contrast values is very difficult to accomplish. Graylevel equalizing these images produces images that have approximately the same brightness and contrast, hence making it easier to compare features within these images. Histogram specification builds upon histogram equalization by providing a means to specify the redistribution of graylevels within an image. ${ }^{3,4}$ With graylevel histogram specification, both the contrast and brightness of the output image can be specified through the desired histogram.

Algorithms have been developed to apply grayscale histogram equalization to color images. $5,6,7,8$ This extension from grayscale to color leads to two problems. First, the pixel values in a grayscale image are scalars, so grayscale histogram equalization is a one-dimensional process. The pixel values in a color image are a vector (usually of red, green, and blue values), which implies that color equalization is a three-dimensional process. If histogram equalization is applied to each of these components independently, changes in the relative percentage of red, green, and blue for each color vector may occur. This modifies the hue of those color vectors, producing color artifacts (undesirable or unnatural colors). Consequently, color equalization must process all three components at once, which makes for a complex histogram and a complex specification process. Faugeras and Strickland have both shown the advantage of using the chromaticity components to process a color image. $^{2,9}$ Faugeras showed that in order for color image processing algorithms to produce the least amount of detectable color distortion, the processed quantities should be mapped as closely as possible to those that are perceptually important. He developed a three-dimensional model for the human visual system based on hue, saturation, and intensity. Strickland et. al. was able to show the importance of the saturation component in the extraction of edge features from a color image. Weeks et. al. used both the saturation and the hue components in the detection of edge features and were able to extract additional edge features by including the hue component in the edge detection process. 1

One common method of histogram equalization of a color image is to treat each of the red, blue, and green components as a graylevel image equalizing each of these independently. The main problem with this method is that it can produce new hue values. that did not exist in the original image, possibly producing unwanted color artifacts. Trahanias and Venetsanopoulos developed a method of color equalization involving 3-D histogram equalization in the RGB color space. ${ }^{8}$ Their method computes the original 3-D histogram for the image and "stretches" it into a uniform distribution. The main limitation with their method is that does not scale well to 24-bit color images requiring $64 \mathrm{MB}$ (4 bytes/cell) for the threedimensional input histogram. Furthermore, adjustment of the RGB components in this way doesn't equalize the saturation of the color vectors within a given hue. Another commonly used method of equalizing a color image is to convert the color image to its achromatic and chromatic components and histogram specify the achromatic component alone. This essentially equalizes the graylevel component of the color image leaving the chromatic components unchanged. However, this method does not use all the information available in the original color image.

Bockstein introduced a method of color equalization that is based on the saturation and luminance components. ${ }^{6}$ He transformed the RGB color space into an HSI color triangle divided into 96 hue regions. He then applied histogram equalization to the saturation component of the color vectors within each of those regions. However, the algorithm is incomplete when determining the maximum possible saturation to use for each transformation. While Bockstein recognized that the maximum saturation is a function of hue, he did not address the fact that it is also a function of luminance. Weeks et. al. improved on Bockstein's method by including both the saturation and the luminance in the color equalization process. 5,7 They recognized the problem that high and low luminance color vectors cannot achieve all possible values of saturation. When this fact is ignored, the resulting transformation produces color vectors which contain "unrealizable" RGB colors (those that map outside the color cube shown in Figure 1(a)). By including the luminance in the equalization process they were able to reduce the number of unrealizable colors that were created. Hence, a color equalized image was produced that contained fewer color artifacts.

Mlsna and Rodriguez presented an algorithm called "histogram explosion."10 One-dimensional histogram processing is done on rays emanating in all directions from a selected vector (called the operating point) to the RGB color space boundary. The primary goal of their algorithm is histogram equalization to improve the contrast of a RGB color image by using the full RGB gamut. In general, with Mlsna and Rodriguez approach, perceptual attributes, such as hue, are not preserved. If the operating point is chosen to be on the grayscale axis, then hue values are preserved. But because the rays on which histogram processing is done emanate in all directions, changes in saturation and luminance are correlated. Furthermore, the 
amount of correlation varies between rays. This makes it difficult to control the other attributes of visual perception (saturation and luminance) in the output image.

A general color histogram specification algorithm must consider the available chromatic information. The selection of the color space in which the image is processed plays a significant role in the algorithm development. This paper presents a new method of color histogram specification that uses the color difference (C-Y) space providing, a means to specify separately hue, saturation, and intensity/luminance histograms while including the interdependency between the individual color components. Since one of the main difficulties associated with histogram specification is determining the required histogram for a desired enhancement, the HSY color model is chosen so that three desired histograms correspond directly with the perceptual color components of hue, saturation, and intensity. Section 2 describes the C-Y color space and the color specification process. Section 3 presents our results and gives an example of using histogram specification on a color image.

\section{COLOR SPECIFICATION}

The color specification algorithm presented in this paper can be implemented using any of the available color models that separate the intensity/luminance from the chromatic information. ${ }^{11}$ There are a number of color spaces which separate the chromatic and achromatic information, and therefore coincide with the physical interpretation of color. The C-Y (color difference) color model is one of these color models and was the one chosen to implement the color histogram specification algorithm presented here. The C-Y color space was chosen for several reasons. The transformations between RGB and HSI are more complex than the corresponding C-Y transformations, increasing the time required to process a color image. ${ }^{2}$ The C-Y color space and the related I-Q color spaces are the standard color spaces chosen by the National Television Standards Committee for color television in the United states. ${ }^{11}$ This makes a real-time hardware implementation of the color histogram specification algorithm feasible. The last reason that the C-Y color space was chosen was that it eliminates the need of using complex equations to obtain the hue and saturation components requiring only trigonometric functions.

Typically, color images are defined in terms of a Red, Green, and Blue component (RGB color model). Each is represented by a graylevel proportional to the component's intensity. The RGB color space is formed by using the red, green, and blue intensities as the three dimensions of a Cartesian coordinate system, as illustrated by Figure 1(a). Also shown are the three primary colors: red, green, and blue. Each of the red, green, and blue color components have 256 distinct intensities, allowing for $\mathbf{1 6 . 7}$ million possible colors. This limits the size of the RGB color space to that of a finite size cube as shown in Figure 1(a). Any specific color can be identified by its particular red, green, and blue graylevels, and is referred to as a color's tristimulus values. Located at six of the eight corners of the RGB cube are the six primary and secondary colors. The line connecting the other two corners (black to white) represents the possible grayscale intensities available in a black and white image.

The main limitation of the RGB color model is that it doesn't correspond to the human visual interpretation of color. The HSI (hue, saturation, intensity) model is one way of representing the red, green, and blue components in terms of chromatic and achromatic information. Figure 1(b) shows the relationship between the RGB cube and the HSI triangle. The HSI triangle is the plane formed by connecting the red, green, and blue corners of the RGB cube. Under the HSI color model, the rectangular red, green, and blue coordinates are transformed into the polar coordinates of hue and saturation. Figure 1(b) isolates the triangle, showing the definitions of hue and saturation as being independent of intensity. Hue is defined as the angular distance between the reference line connecting the center of the triangle and the color red. Saturation represents how much white is present in a color and is measured as the radial distance from the center of the triangle to the given color point. Low saturation colors (pastels) are located at the center of the triangle while the boundary contains the highly saturated colors.

Figure 2(a) shows the C-Y color axes $(R-Y$ and $B-Y)$, and the definition of hue $(\theta)$, and saturation $(S)$. Transforming the RGB color space into the C-Y space scales the cube and rotates it such that the "white" corner is in line with the origin of the $\mathrm{C}-\mathrm{Y}$ space. This is shown in Figure 2(b). The transformation to obtain the $\mathrm{C}-\mathrm{Y}$ components $Y, R-Y$, and $B-Y$ is: 


$$
\left|\begin{array}{c}
Y \\
R-Y \\
B-Y
\end{array}\right|=\left|\begin{array}{rrr}
0.299 & 0.587 & 0.114 \\
0.701 & -0.587 & -0.114 \\
-0.299 & -0.587 & 0.886
\end{array}\right|\left|\begin{array}{c}
R \\
B \\
G
\end{array}\right| \text {, }
$$

where $R, G$, and $B$ are the red, green, and blue components of the RGB color, $Y$ is the luminance, and $R-Y$ and $B-Y$ specify the chromaticity. The unequal weighting of red, green, and blue in the luminance calculation reflects the differences in perceived brightness for each of the RGB component colors.

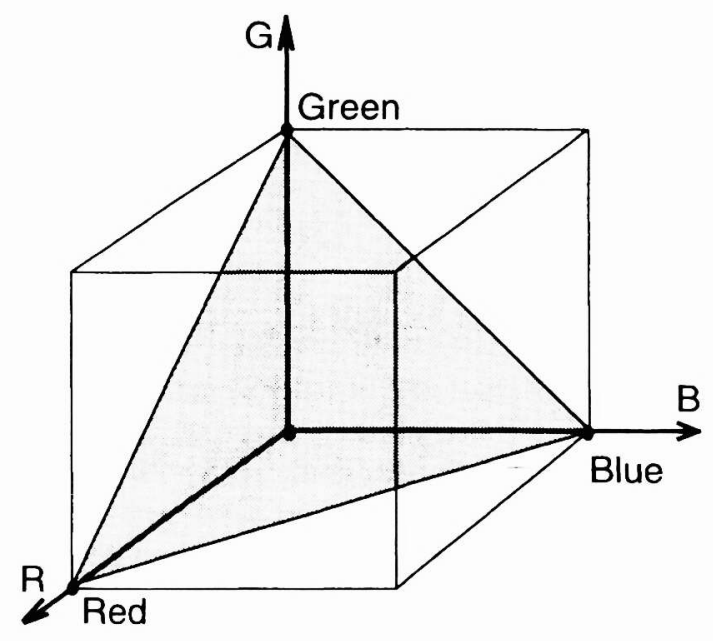

(a)

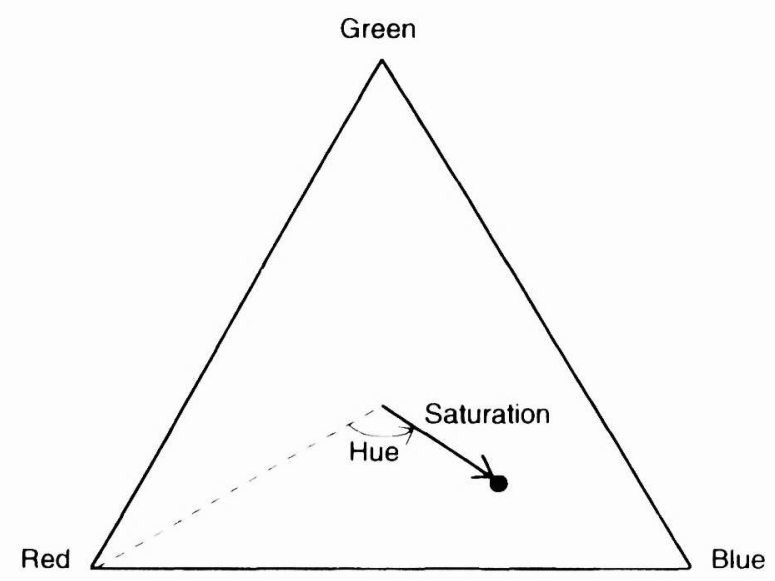

(b)

Figure 1: (a) The relationship between the RGB cube and the HSI triangle and (b) the HSI color triangle showing hue and saturation.

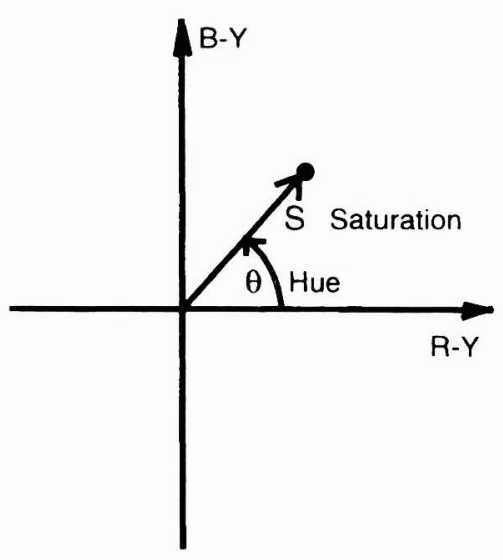

(a)

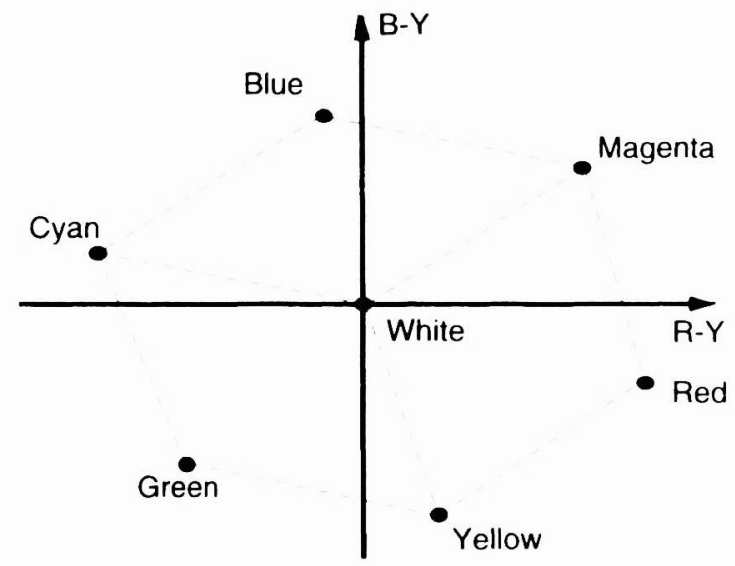

(b)

Figure 2: (a) The C-Y plane, defining hue and saturation and (b) mapping of the RGB cube to the $\mathrm{C}-\mathrm{Y}$ space.

Once in the C-Y plane, the chrominance can be represented by the rectangular coordinates $R-Y$ and $B-Y$, or the polar coordinates of hue and saturation. The conversions between the two are accomplished using standard rectangular-to-polar equations: 


$$
\theta=\tan ^{-1}\left[\frac{B-Y}{R-Y}\right]
$$

and

$$
S=\sqrt{(R-Y)^{2}+(B-Y)^{2}},
$$

where the saturation $S$ is the length of the line from the origin to the color of interest, and hue $\theta$ is the angle between the $R-Y$ axis and the saturation line, as defined in Figure 2(a). The inverse transformation obtains the red, green, and blue components by adding the luminance value $Y$ to $R-Y, B-Y$, and $G-Y$ according to:

$$
\begin{aligned}
& R=S \sin \theta+Y \\
& B=S \cos \theta+Y \\
& G=-0.51(R-Y)-0.19(B-Y)+Y .
\end{aligned}
$$

Only $R-Y$ and $B-Y$ components are required, since the $G-Y$ component can be expressed in terms of $R-Y$ and $B-Y$.

There are several steps involved in applying histogram specification to a color image. The direct approach to color histogram specification requires specification of a three-dimensional joint RGB histogram. Given the desired threedimensional histogram representing the three-dimensional RGB color vectors, this histogram is equalized generating a threedimensional mapping function $y=g(z)$ between the desired histogram and an intermediate equalized histogram ( $z$ is a color vector representing the specified histogram that maps to a color vector $y$ in an equalized intermediate histogram). Next, the original color image is also equalized, generating a mapping function $\boldsymbol{w}=f(\boldsymbol{x})$ between the original image's histogram and an intermediate equalized histogram. ( $x$ is a color vector in the original image that maps to color vector $w$ in the equalized intermediate image.) Since the histogram of the equalized original image and the equalized desired histogram are both uniformly distributed, then $y$ and $w$ can be equated: $g(z)=y=w=f(x)$. Solving for the $z$ yields the final mapping function $z=g^{-1}(f(x))$ that produces a new color image whose distribution matches the desired histogram. Unfortunately, this direct approach of using a color's RGB values is very computationally complex requiring a large amount of memory. Furthermore, this approach gives very little insight to the type of three-dimensional distribution that is required for a particular enhancement. Typically, for graylevel histogram specification, the desired histogram is chosen based upon the type of graylevel enhancement that is desired. The goal of histogram specification for a color image is to select a set of specified histograms that corresponds to a desired enhancement.

Since the human visual system interprets a color as three independent components of hue, saturation, and luminance, a better approach is to transform the color image from the RGB color space using the HSY color space using Equations (1) (3). Then the joint distribution $p(Y, \theta, S)$ can then be written as

$$
p(Y, \theta, S)=p(S) \cdot p(\dot{\theta}) \cdot p(Y)
$$

Equation (5) reduces the color histogram specification problem from one three-dimensional histogram specification to three independent one-dimensional histogram specifications (one for each of the $H, S$, and $Y$ color components). An advantage to this approach is that the effect that each specified distribution has on the resulting image is easily identified. Unfortunately, Equation (5) describes the ideal case. Converting a 24-bit RGB color image to its HSY color space representation and then appling Equation (5) directly would result in colors exceeding the finite limits of the original RGB color space. This occurs when the HSY color space representation is converted back to its original RGB representation. Hence, this limits the distribution of color throughout the RGB color space. The work of Weeks et. al. ${ }^{5,7}$ has shown that the maximum saturation that can be achieved in a finite color space depends on the luminance and hue. For example, the RGB colors of (255, 255, $255)$ as well as $(0,0,0)$ can only have a saturation of zero. Therefore, the correct distribution for saturation is the conditional distribution $p(S \mid Y, \theta)$. If this is not considered, then a large number of color vectors will be moved outside the realizable space and subsequently clipped when converted back to RGB resulting in color artifacts. The three-dimensional distribution that represents the correlation between the saturation and the luminance color components is given by: 


$$
p(y, \theta, S)=p(S \mid Y, \theta) \cdot p(\theta) \cdot p(Y)
$$

If the desired distribution for the saturation is the same shape for each luminance and hue region, then this saturation distribution is nothing more than a scaled version at each luminance $p(s)=p(s, \operatorname{smax}(Y, \theta))$, where $\operatorname{smax}(Y, \theta)$ is the maximum saturation at luminance $Y$ and hue $\theta$. A unique distribution can be specified for each luminance/hue region, or for each hue region (same distribution shape for a particular hue at all luminance levels). If it is assumed that the shape of $p(S)$ $Y, \theta)$ is the same for each luminance and hue, then a single histogram can be specified that is parameterized on the maximum saturation value for the corresponding luminance/hue region. Another variation is to allow the hue distribution to be dependent on the luminance level:

$$
p(Y, \theta, S)=p(S \mid Y, \theta) \cdot p(\theta \mid Y) \cdot p(Y)
$$

Equation (7) describes the most flexible of the three approaches, yet it is the most difficult to implement. Whereas the conditional distribution of saturation was required because of a finite color space, this conditional distribution of hue is not due to a limited color space but only provides more flexibility for the user. Fortunately, most histogram specification applications do not alter the hue component, but modify only the saturation and luminance components. This allows Equation (6) to be used successfully.

\section{RESULTS}

The color specification algorithm was implemented on a desktop computer using the $\mathrm{C}++$ programming language. Equation (6) was implemented, whereby all the pixel values in an image are used to produce the desired luminance and hue distributions. Histogram specification was first performed on the luminance, and then on the hue if desired. Saturation was then distributed conditioned on luminance, hue, and maximum saturation for the given luminance and hue. 64 luminance regions and 16 hue regions were used, splitting the $C$-Y color space into 1024 regions. A $64 \times 16$ array holds the maximum saturation value for each region. Only the color vectors within a region are used to match the desired saturation histogram for that region: $p(S \mid Y, \theta)$. This allows 1024 unique saturation histograms to be specified. We allowed specification of sixteen different saturation histograms based on hue. Within each hue region, 64 specified histograms were generated and then scaled by the corresponding maximum saturation for the luminance/hue region. For example, the 64 saturation histograms for the red hue region (one for each luminance region) are the same shape, but are of different widths due to the varying maximum saturation.

The absence of an absolute ordering for the hue component also hinders the specification of an arbitrary histogram for hue. A hue of zero degrees lies somewhere in the red region; some red hues are slightly greater than zero, and others are close to $2 \pi$. It may be desired to shift the red hues closer to another hue, say green. But because histogram processing does not alter the ordering of pixels, and the red hues appear at both ends of the hue range, the red hues will not be shifted as a group. The desired histogram shape may be produced, but without the desired effect. To overcome this problem, we allow the base hue angle to be specified. This is the angle at which the hue coordinate is split for histogram processing. In the previous example, if the base angle is specified as a blue hue, then the red hues do appear as a group in the histogram and will maintain the appropriate ordering.

The example image presented in this paper is that of a woman in an airport (Figure 3) that was obtained with uneven illumination. The woman's face and clothing (foreground) are well lit, but the background is dark. Figure 4 shows a plot of the C-Y chromatic plane with the R-Y axis along the horizontal direction (as viewed from the positive luminance axis). This plot does not represent the density of color vectors; it simply shows where color vectors are found in the C-Y color plane. When color vectors map onto the same B-Y/R-Y coordinate, the color vector with the higher luminance is shown in the plot; so the brightness of the C-Y plot represents the brightest color vectors. It is apparent from this C-Y plot that the original image contain hues that are a predominantly blue/purple (the woman's skirt) and red/orange (flesh tones). Overall this image is a low saturated image.

Figure 5 shows the airport image after luminance equalization only. Features in the background undetectable in the original are now apparent: e.g., the man, the benches, and the windows and column. However, the background appears 


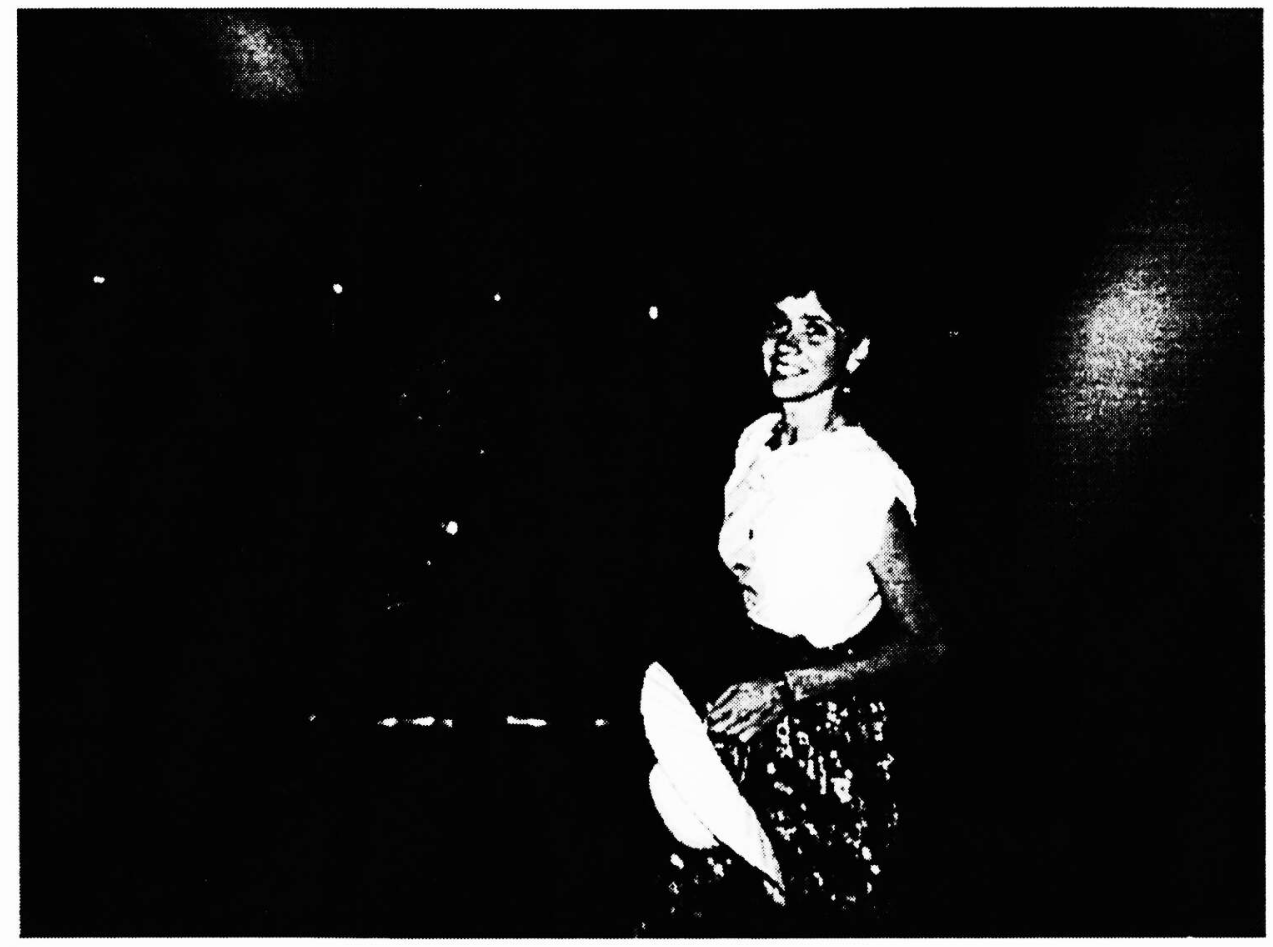

Figure 3: An example 24-bit color image of a woman in an airport with uneven illumination

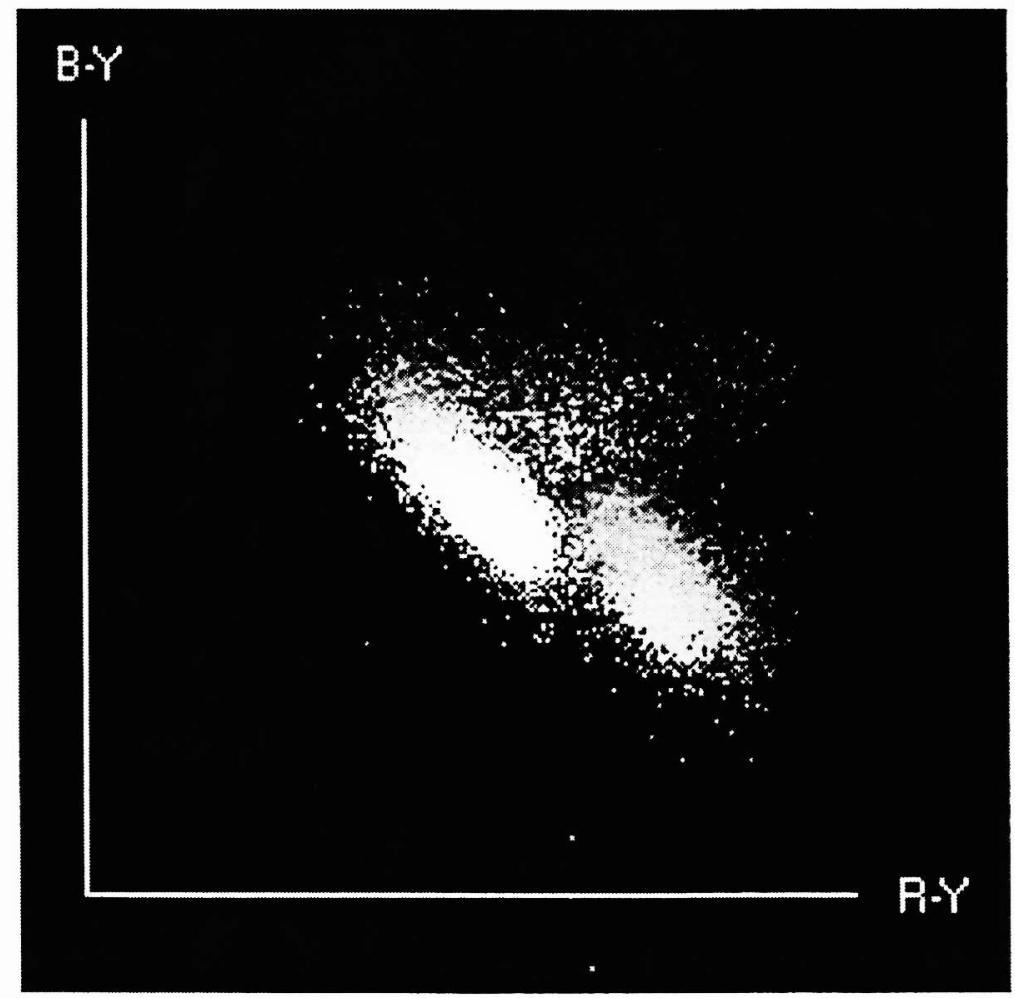

Figure 4: A plot of the C-Y chromatic plane with the R-Y axis along the horizontal direction. 


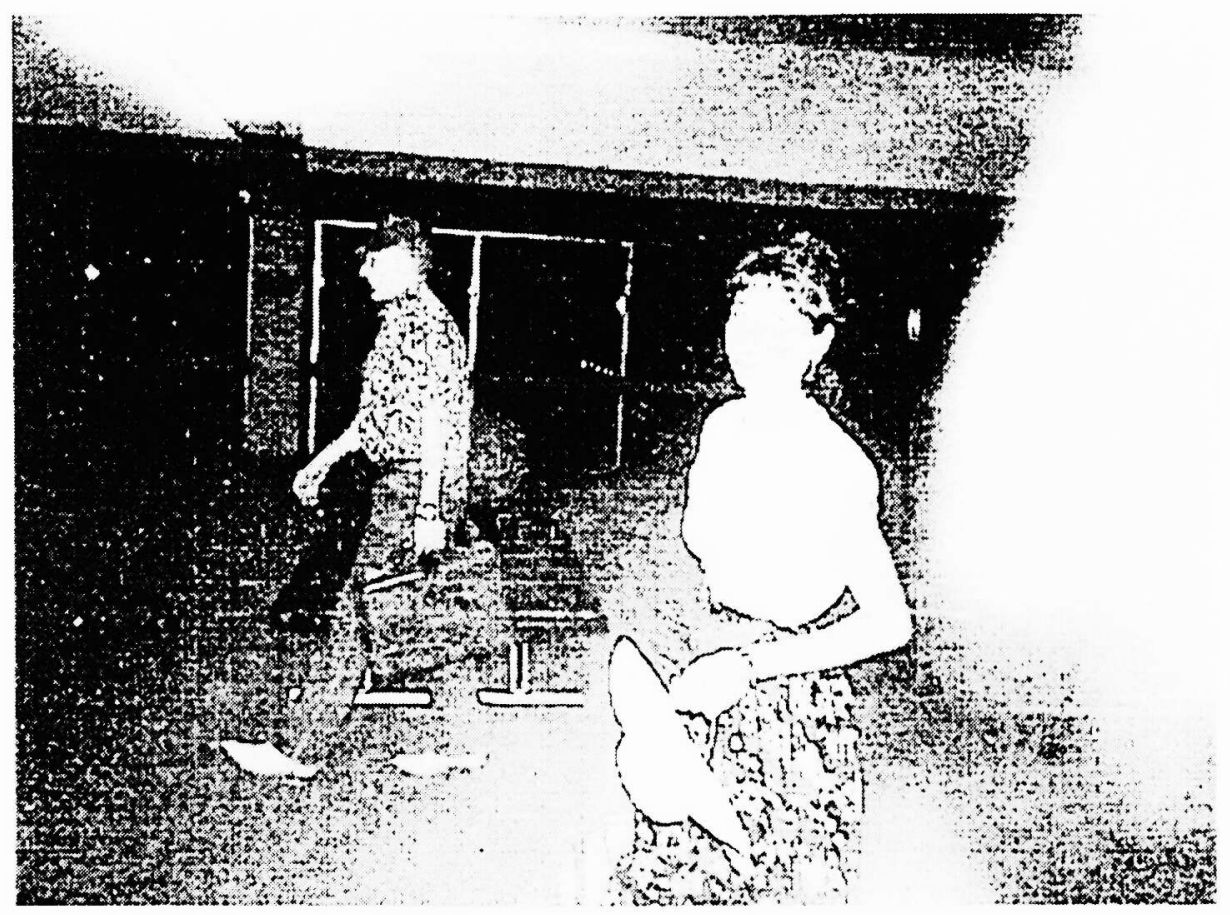

Figure 5: Luminance only equalization of Figure 3.

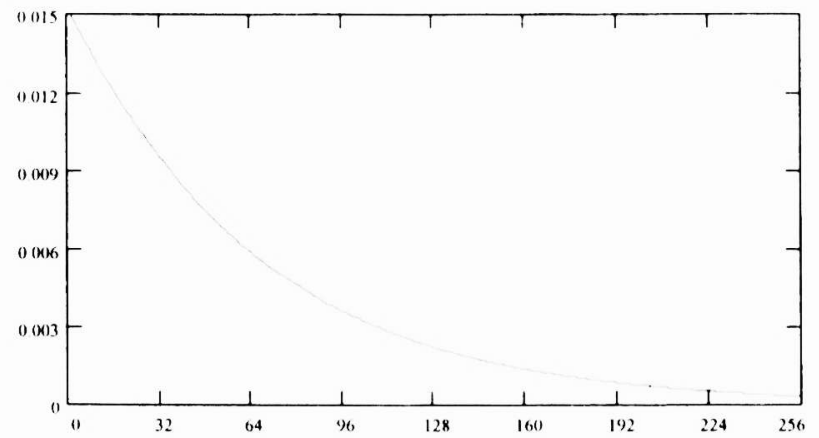

(a)

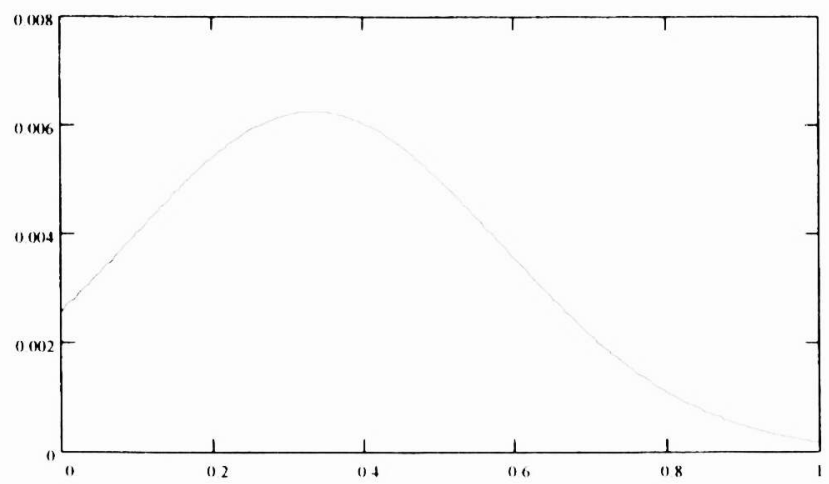

(b)

Figure 6: (a) The desired histograms for the luminance component and (b) for the saturation component within each each luminance/hue region. 


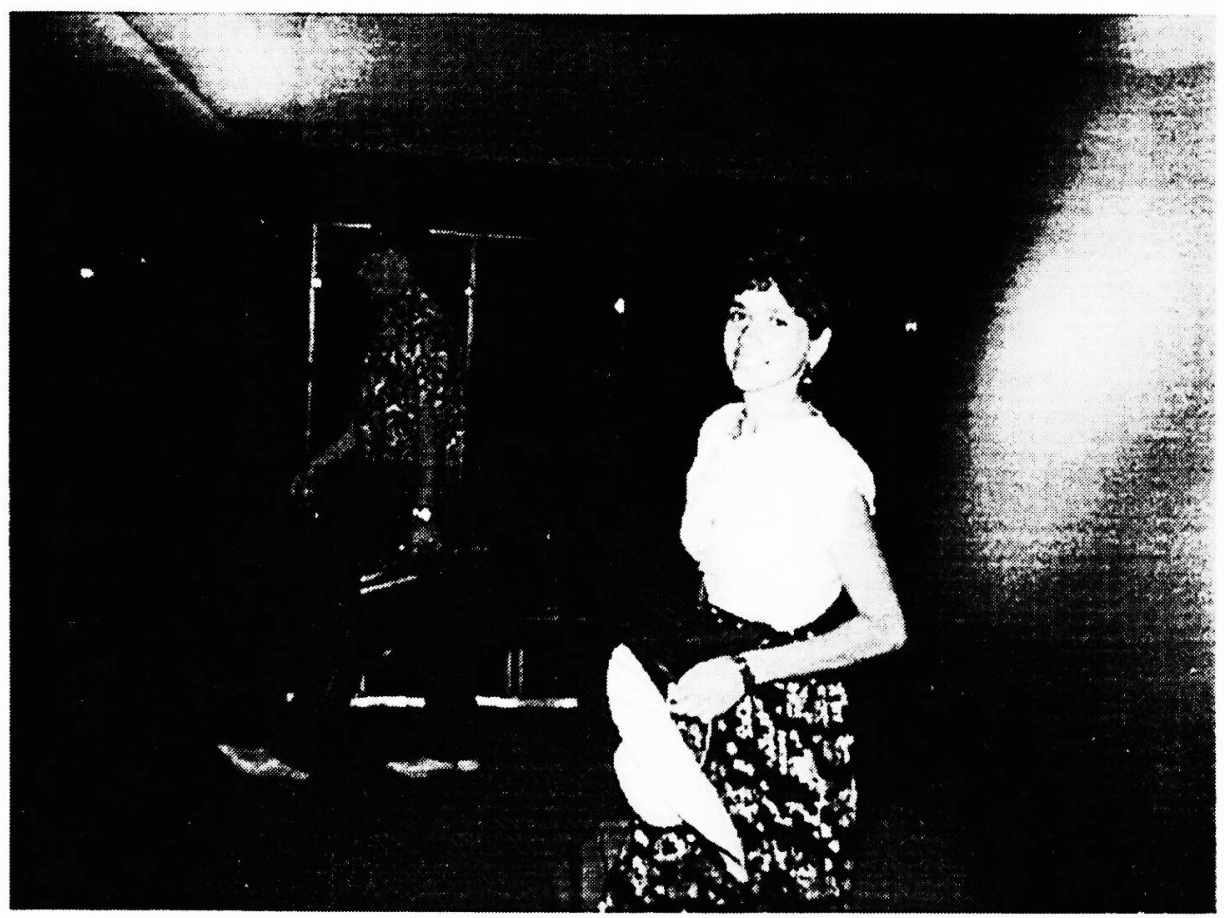

(a)

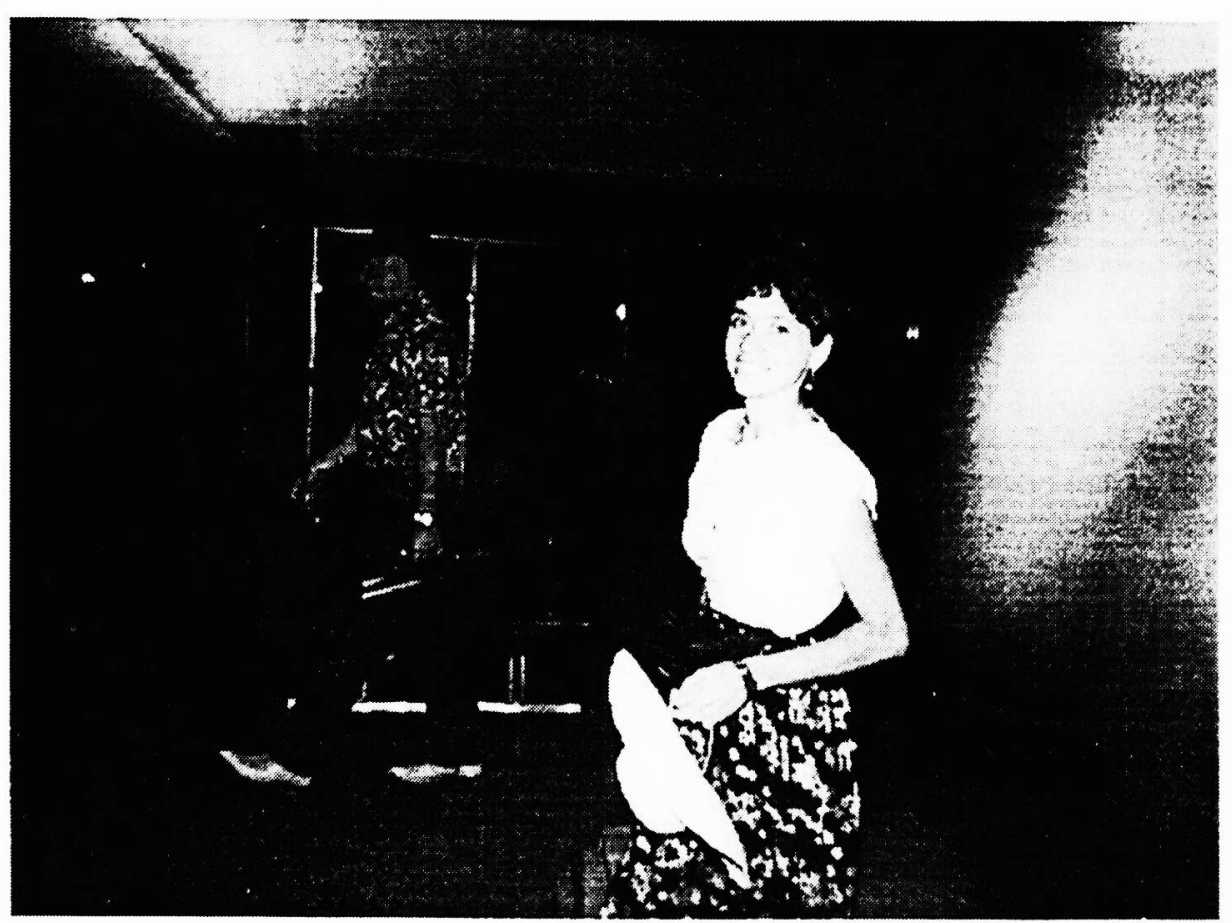

(b)

Figure 7: (a) The histogram specified image obtained with the luminance and saturation distributions given in Figure 6 and (b) the histogram specified image obtained with the same luminance and saturation distributions given in Figure 6 but with the saturation distribution applied only to the blue hue regions. 


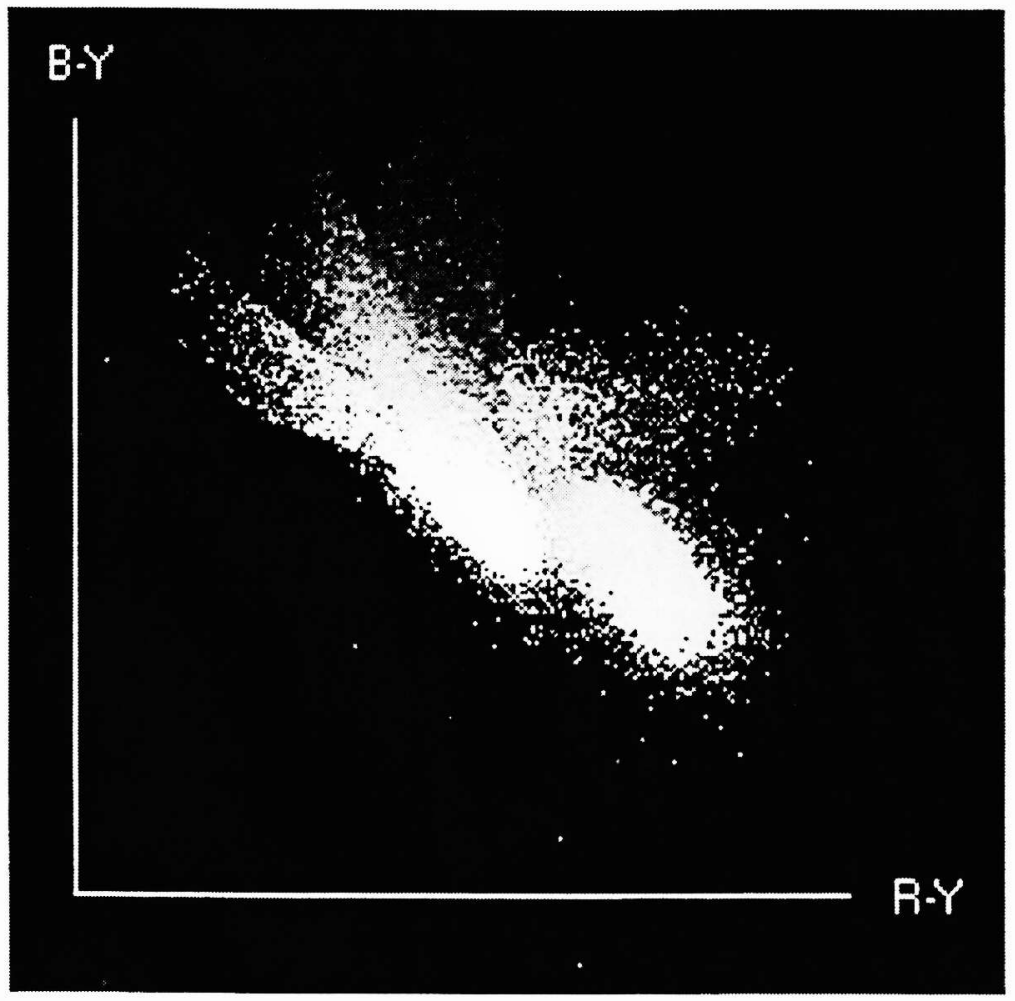

Figure 8: The C-Y plot for the histogram specified image given in Figure 7(b).

blotchy and the woman is now over-saturated. The original airport image was then enhanced by specifying a normalized negative exponential distribution

$$
p(x)=c \cdot e^{-0015 x},
$$

for the luminance (Figure 6(a)) and a Gaussian as the saturation distribution within each luminance/hue region (Figure $6(\mathrm{~b})$ ). The enhanced image using this luminance distribution and this saturation distribution is shown in Figure 7(a). Notice that the blue objects (man's shirt and jeans, and the woman's skirt) are enhanced, but other color objects are overly-saturated (flesh tones too red, hat and shirt have green tint, briefcase has red blotches). The final enhanced image (Figure 7(b)) was obtained using the same luminance distribution and same saturation distribution, but the saturation distribution was only applied to the luminance/hue regions with blue hues. This image reveals objects not visible in the original, yet maintains a natural appearance. The C-Y plot for this image is shown in Figure 8; notice how the blue hues have been stretched, but the other hues maintain the same shape as in the original $\mathrm{C}$-Y plot.

\section{CONCLUSIONS}

The color histogram specification method presented in this paper extends the color histogram equalization presented by Weeks. et.al. ${ }^{5.7}$ Histogram specification was able to enhance images while reducing the addition of objectionable colors and hue noise as compared to histogram equalization. Specifying a saturation distribution dependent on hue proved beneficial in enhancing specific image details without degrading others. Color histogram specification is more effective when performed in a perceptual color space, such as the C-Y color space, than in the RGB space. As a final note, electronic versions of the color images presented in this paper are available by contacting the authors. 


\section{REFERENCES}

1. A. R. Weeks, C. E. Felix and H. R. Myler, "Edge Detection of Color Images using the HSI color space", IS\&T/SPIE conference, San Jose, February, 1995.

2. R. N. Strickland, C. S. Kim, and W. F. McDonnel, "Digital color image enhancement based on the saturation component," Optical Engineering, Vol. 26, No. 7, pp. 609-616, 1987.

3. A. R. Weeks, Fundamentals of Electronic Image Processing, pp. 109-120, 228-293, SPIE Press, Bellingham, Wa. (1996).

4. R. C. Gonzalez and R. E. Woods, Digital Image Processing, Addison-Wesley, Reading, MA, pp. 137-180, 227-229, 247-248, 1992.

5. G. E. Hague, A. R. Weeks, and H. R. Myler, "Histogram Equalization of the Saturation Component for True-Color Images using the C-Y Color Space," SPIE: San Diego, July, 1994.

6. I. M. Bockstein, "Color equalization method and its application to color image processing," J. opt. Soc. Am. A, Vol. 3, No. 5, pp. 735-737 (1986).

7. A. R. Weeks, G.E. Hague, and H.R. Myler, "Histogram equalization of 24-bit color images in the color difference (C-Y) color space," J. Elec. Imaging 4(1), pp. 15-22, (January 1995).

8. P. E. Trahanias and A. N. Venetsanopoulos, "Color image Enhancement Through 3-D Histogram Equalization," Proceedings of the 15th IAPR International Conference on Pattern Recognition, The Hague, Netherlands, pp. 545-548 (August 1992).

9. O. D. Faugeras, "Digital Color Image Processing Within the Framework of a Human Visual Model," IEEE Trans. Acoust., Speech, Sig. Proc. ASSP-27 (4), pp. 380-393 (1979).

10. P. A. Mlsna and J. J. Rodriquez, "A Multivariate Contrast Enhancement Technique for Multi-spectral Images," IEEE Trans. on Geoscience and Remote Sensing, 33(1), pp. 212-216 (January 1995).

11. A. V. J. Martin, Technical Television, Prentice-Hall Inc., Englewood Cliffs, NJ, pp. 498-503 (1962). 